\title{
Manejo de fractura de cuello femoral en paciente con amputación supracondílea femoral bilateral y revisión de la literatura
}

\author{
Femoral neck fracture management in patient with bilateral femoral \\ supracondylar amputation and literature review \\ Martínez-Hernández A,* Nieto-Alvarado S \\ Centenario Hospital Miguel Hidalgo.
}

\begin{abstract}
RESUMEN. El tratamiento de un paciente con amputación supracondílea femoral bilateral, y que ha sufrido una fractura de cuello femoral, es un evento poco común tanto para el cirujano ortopédico como para el equipo de rehabilitación. Presentamos un caso, en el cual se discuten diferentes dificultades en su tratamiento, elección de implante y regreso a sus actividades diarias. Se trata de un joven con diagnóstico de amputación traumática bilateral supracondílea, con fractura de cuello femoral, tratado mediante artroplastía total de cadera primaria no cementada. La evaluación de la funcionalidad de la prótesis total de cadera con escala de Houghton fue al cabo de 12 meses, se obtuvo una puntuación final de 9 puntos, los cuales son el resultado de una rehabilitación satisfactoria.
\end{abstract}

Palabras clave: Artroplastía total de cadera, amputación supracondílea femoral bilateral, fractura de cuello femoral.

\section{Introducción}

En Estados Unidos aproximadamente 1.6 millones de personas han tenido una amputación de la extremidad inferior y se tiene una tasa de incidencia de 30,000 a 40,000 amputaciones de extremidades inferiores que se realizan anualmente. ${ }^{1}$ La mayoría de las amputaciones de extremidades inferiores se realizan por complicaciones de enfermedades vasculares periféricas, diabetes mellitus o ambos, trauma en las extremidades inferiores, tumores musculoes-
ABSTRACT. The treatment of a patient with amputation above the knee who has suffered a femoral neck fracture is a challenge for both the orthopedic surgeon and the rehabilitation team. We present a case, in which different difficulties are discussed in their treatment, choice of implant and return to their daily activities. The clinical case of a young man diagnosed with supracondylar bilateral traumatic amputation, with fracture of the femoral neck, treated by total non-cemented hip arthroplasty is presented. The functionality of the total hip prosthesis with Houghton scale after 12 months, obtaining a final score of 9 points which are the result of a satisfactory rehabilitation.

Keywords: Total hip arthroplasty, bilateral supracondylar amputation, femoral neck fracture.

\footnotetext{
${ }^{\ddagger}$ Médico residente de cuarto año de Traumatología y Ortopedia.

Centro Hospitalario Miguel Hidalgo.

Dirección para correspondencia:

Dr. Ángel Martínez Hernández

Avenida Gómez Morín s/n, Alameda, CP. 20259, Aguascalientes, Ags.

Tel: 449-448-62-57

E-mail: angelmtz-dd@hotmail.com
}

* Jefe del Departamento de Traumatología y Ortopedia. Jefe del Servicio de Cirugía Articular y Artroscópica.

https://dx.doi.org/10.35366/93351

doi: $10.35366 / 93351$

queléticos, infección o sepsis y etiologías congénitas representan el resto de las amputaciones en las extremidades inferiores. ${ }^{1}$

La fractura del cuello del fémur en un paciente con amputación es una entidad poco común de admisión en unidades de traumatología. ${ }^{1}$ Salai tiene la serie de pacientes más grande con cinco pacientes con amputación infrageniana de miembro inferior, intervenidos de artroplastía total de cadera por fracturas subcapitales de fémur desplazadas, con resultados funcionales alentadores. ${ }^{2,3}$ Por 

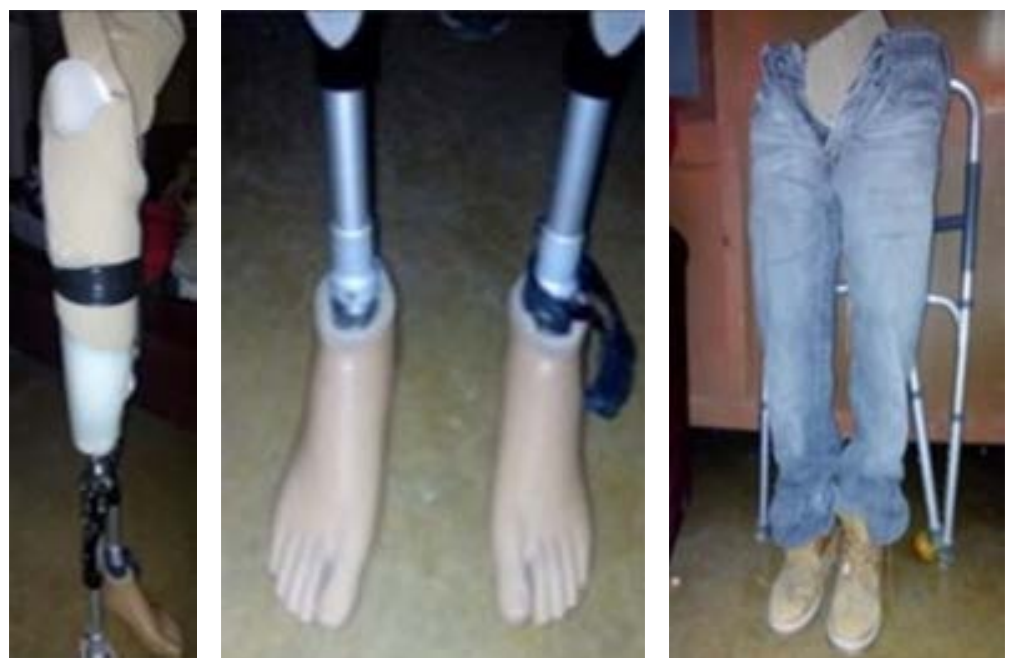

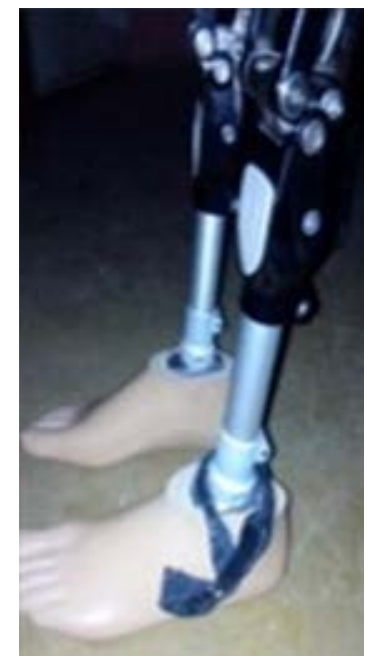

Figura 1:

Exoprótesis bilateral del paciente. otro lado, Nejat y colegas, en el año 2005, presentaron en su revisión dos casos de pacientes con artroplastía total de cadera sobre amputaciones de miembro inferior ipsilateral, una suprageniana ipsilateral y otro sobre amputación de Syme.

Todo paciente con amputación presenta deficiencias musculares, generación de osteoartritis severa, la cual aumenta hasta $61 \%$, así como osteoporosis, que al utilizar exoprótesis generará un brazo de palanca importante generando fracturas de cadera. ${ }^{1,2}$

Algunos autores defienden el tratamiento conservador en pacientes amputados con fracturas de cadera o reducción y fijación interna, así como artrodesis de cadera; sin embargo, el riesgo de no unión o de fractura es muy alta. ${ }^{1}$ El reemplazo articular es una buena opción en pacientes con fractura de cuello femoral mejorando su pronóstico, con una alta tasa de supervivencia y resultados funcionales satisfactorios en comparación con el manejo conservador. $2,3,4,5,6,7,8$

Se reporta el segundo caso de fractura del cuello femoral en un paciente con amputación bilateral supracondílea por traumatismo, tratada con artroplastía total primaria no cementada de cadera. ${ }^{9}$

\section{Caso clínico}

Masculino de 31 años de edad, con amputación supracondílea femoral bilateral de extremidades inferiores por traumatismo laboral hace dos años. El paciente deambulaba con uso de exoprótesis y con apoyo de andadera (Figura 1), se presentó en la consulta externa de pelvis y cadera en el departamento de nuestra institución por presentar dolor de cadera derecha de un mes de evolución que no cede con analgésicos, así como dificultad para su movilización de la misma extremidad, se refiere con antecedente de sufrir múltiples caídas de propia altura.

Se solicitaron radiografías iniciales. Se tomó una radiografía anteroposterior de pelvis que reveló un trazo de fractura subcapital del cuello femoral derecho, Garden IV (Figura 2).
Se ofreció al paciente el tratamiento quirúrgico con una artroplastía total primaria de cadera (ATC) no cementada y el paciente estuvo de acuerdo con el procedimiento.

La cirugía se realizó con un tiempo quirúrgico de dos horas 30 minutos y un sangrado de $1,200 \mathrm{~cm}^{3}$, abordaje lateral directo, presentó múltiples dificultades como posicionamiento, control rotacional y tracción para colocación de componente femoral y posterior tracción y rotación interna para reducir prótesis. De la literatura descrita sólo se utilizó un clamp para control rotacional. El resto de la cirugía cursó sin complicaciones. Se colocó drenovac de $1 / 4$, el cual se dejó 24 horas con un tiempo de estancia intrahospitalaria de 48 horas, su manejo farmacológico consistió en ketorolaco $30 \mathrm{mg}$ cada ocho horas, enoxaparina $60 \mathrm{mg}$ intravenosa cada 24 horas y terapia antimicrobiana con ceftriaxona 1 gramo intravenosa cada 12 horas. Por otro lado, se inició rehabilitación pasiva. A su egreso se dejó tratamiento con cefalexina 500 mg vía oral cada ocho horas por siete días,

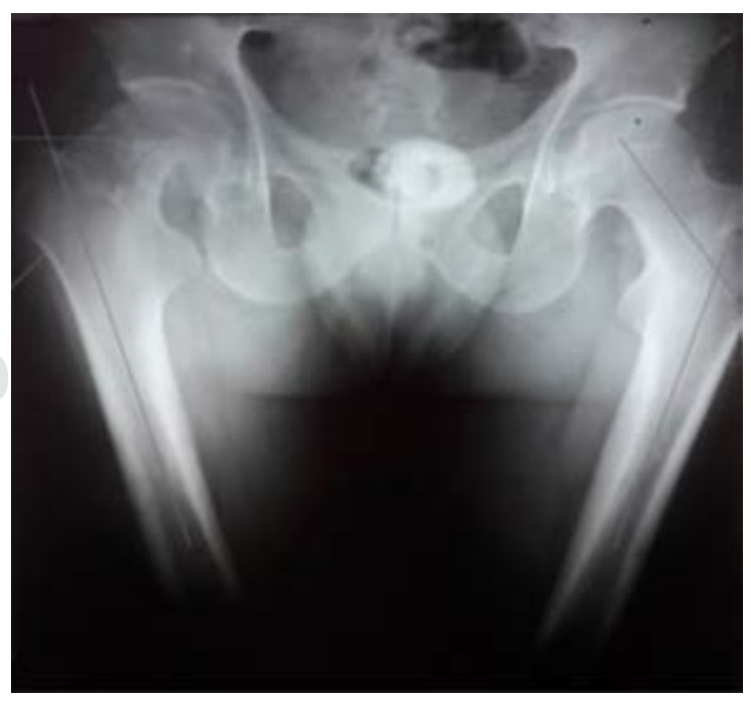

Figura 2: Radiografía anteroposterior de pelvis prequirúrgica, evidencia de fractura subcapital derecha. 
dabigatrán por 21 días, ketoprofeno 100 mg vía oral cada 12 horas. Los controles radiológicos de la cadera derecha fueron con medición de offset 46.7 y boost $1 \mathrm{~mm}$ (Figura 3). Se dio de alta con citas subsecuentes a las dos, cuatro y ocho semanas con posteriores revisiones semestralmente.

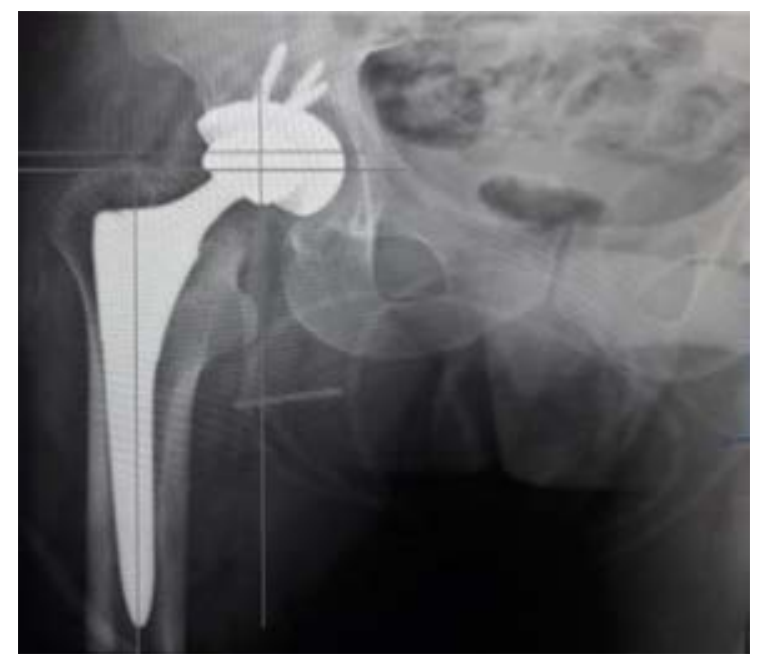

Figura 3: Radiografía postquirúrgica. Offset 46.7 y boost $1 \mathrm{~mm}$.
Su cita a las dos semanas mostró herida en buenas condiciones, sin presentar datos de infección, por lo que se inició readaptación de la marcha con carga de peso completo a los cuatro meses con apoyo de andadera con uso de exoprótesis. Evaluamos la funcionalidad de prótesis externas en amputados con escala de Houghton ${ }^{10}$ al cabo de cuatro, ocho y 12 meses (Tabla 1), obteniendo una puntuación final de 9 puntos, calificando como independiente para sus desplazamientos (Figura 4).

\section{Discusión}

En nuestro caso elegimos un reemplazo total de cadera debido a la edad relativamente joven del paciente (33 años), el alto nivel de actividad y autonomía utilizando sus prótesis habituales, así como la alta esperanza de vida (sin historial de enfermedades cardiovasculares o respiratorias). Kandel realizó un tratamiento a base de una hemiartroplastía bipolar con pronta rehabilitación y readaptación de su estilo de vida. ${ }^{7}$

Se plantean varios problemas en la colocación de una prótesis de cadera como son: un brazo de palanca corto con dificultades para manejar el fémur proximal durante el retiro de la cabeza femoral e inserción de implantes. Algunos

Tabla 1: Escala de Houghton resultados funcionales cuatro, ocho y 12 meses.

\begin{tabular}{|c|c|c|c|c|}
\hline & \multicolumn{4}{|c|}{ Instrumento de Houghton } \\
\hline & & 4 meses & 8 meses & 12 meses \\
\hline \multicolumn{5}{|l|}{ 1. El paciente utiliza la prótesis para desplazarse } \\
\hline a) $<25 \%$ de su deambulación & 0 & $\mathrm{X}$ & & \\
\hline b) $25-50 \%$ de su deambulación & 1 & & $\mathrm{X}$ & \\
\hline c) > 50\% de su deambulación & 2 & & & $\mathrm{X}$ \\
\hline d) durante todos los desplazamientos & 3 & & & \\
\hline \multicolumn{5}{|l|}{ 2. El paciente utiliza su prótesis para andar } \\
\hline a) Solamente para las visitas a rehabilitación & 0 & $\mathrm{X}$ & & \\
\hline b) En casa, pero no para salir al exterior & 1 & & $\mathrm{X}$ & \\
\hline c) Ocasionalmente en el exterior de la casa & 2 & & & \\
\hline d) En casa y en el exterior todo el tiempo & & & & $\mathrm{X}$ \\
\hline \multicolumn{5}{|l|}{$\begin{array}{l}\text { 3. Cuando el paciente camina fuera de casa con su } \\
\text { prótesis }\end{array}$} \\
\hline a) Utiliza una silla de ruedas & 1 & $\mathrm{X}$ & & \\
\hline b) Utiliza dos bastones, dos muletas o andador & 2 & & $\mathrm{X}$ & $\mathrm{X}$ \\
\hline c) Utiliza bastón & 3 & & & \\
\hline d) No necesita ayuda & 4 & & & \\
\hline \multicolumn{5}{|l|}{$\begin{array}{l}\text { 4. Cuando el paciente camina en el exterior con su } \\
\text { prótesis, se siente inestable }\end{array}$} \\
\hline a) En la marcha por terreno llano & 1 & $\mathrm{X}$ & $\mathrm{X}$ & \\
\hline b) En la marcha por pendientes & 2 & & & $\mathrm{X}$ \\
\hline c) En la marcha por terreno irregular & 3 & & & \\
\hline d) En las tres anteriores & 4 & & & \\
\hline $\begin{array}{l}\text { Máxima puntuación: } 12 \text { puntos } \\
9 \text { puntos satisfactoria rehabilitación }\end{array}$ & & 2 & 5 & 9 \\
\hline
\end{tabular}



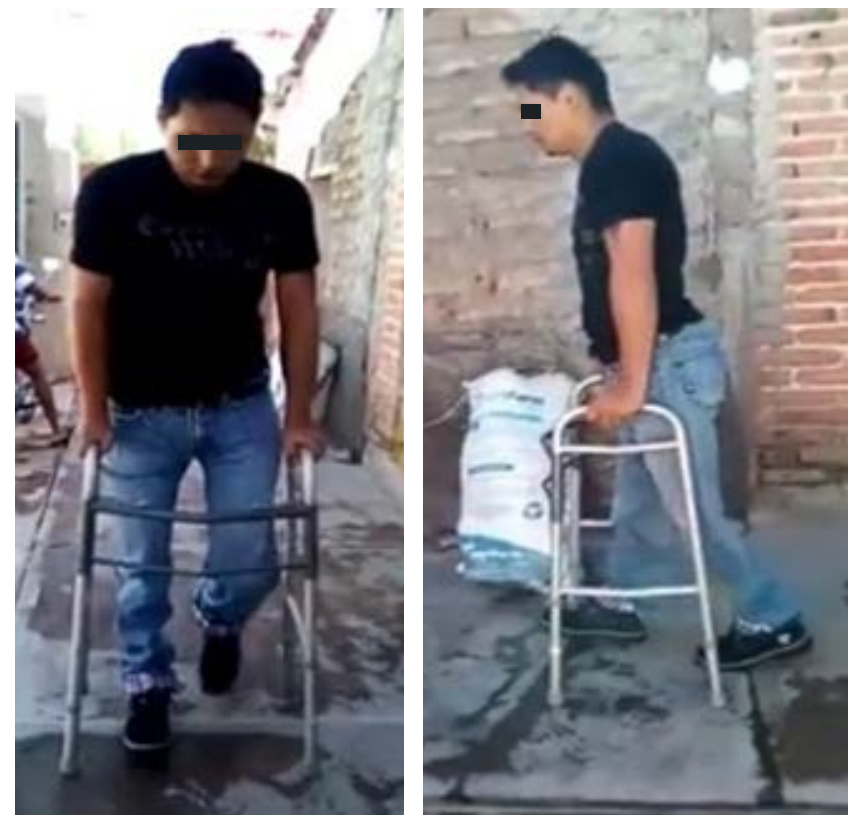

Figura 4: Resultados a los 12 meses de operado.

cirujanos recurrieron al uso de un pin de tracción con una abrazadera ósea o un soporte óseo en el área subtrocantérica para tener un control rotatorio y de tracción satisfactorio en el muñón. ${ }^{2}$

Un gran problema para la manipulación es la contractura de los flexores de la cadera y los abductores que pueden requerir liberación previo a la colocación de componentes protésicos.

Para nuestro conocimiento, el presente caso representa el segundo reportado de un paciente que se sometió con éxito a una ATC después de la amputación bilateral traumática y fractura de cuello femoral. ${ }^{9}$

Durante nuestro procedimiento quirúrgico las principales dificultades enfrentadas están relacionadas con la manipulación femoral y la realización precisa de antisepsia, por lo cual debemos anticipar la posibilidad de mejorar la manipulación que resolverán las dificultades que vamos a tener, recomendamos el aislamiento del muñón con un plástico y tela adhesiva durante el tiempo del procedimiento para aislar la parte distal de éste, que presumiblemente está contaminada, debido a su inclusión habitual en el receptáculo de la exoprótesis y a la irregularidad de su cicatriz. El posicionamiento correcto del implante es esencial para un buen resultado después de una ATC. En pacientes amputados no se tienen las referencias anatómicas comunes, como son la zona condilar interna o el eje de la pierna que ayudan con la anteroversión y dirección del componente femoral. Prickett y Scanlon ${ }^{11}$ informaron una retroversión inserción del vástago femoral en un paciente con amputación femoral. En los casos de ATC, el riesgo de mala posición es aún mayor; en tal situación, la prótesis externa debe ajustarse para enmendar la alteración de la extremidad inferior. ${ }^{9}$ Sin embargo, esta situación no es deseable porque conduce a un mayor riesgo de luxación, la supervivencia de los componentes estarán comprometidos y la cinemática de la marcha alterada. ${ }^{12,13}$

También recomendamos tener un equipo esterilizado de clavos de diferentes medidas listo, lo cual permitirá la inserción de un pin en la diáfisis que se puede conectar a una tracción ósea temporal que ayudará con la buena manipulación del muslo. Turney y colaboradores demostraron que los pacientes con amputación supracondílea generalmente obtienen una mejor movilidad que otros $(\mathrm{p}=0.002)$. La movilización postoperatoria con o sin muletas deben tenerse en cuenta para predecir el resultado en un paciente amputado con un ipsilateral ATC. ${ }^{12}$

Debemos desarrollar un programa de rehabilitación enfocado en la cadera a fin de evitar contracturas, mantener un régimen centrado en el fortalecimiento de los flexores de la cadera y los extensores de la rodilla, los cuales son necesarios para evitar la contractura de flexión de la misma, tanto como sea posible. Durante la cirugía se realiza liberación de partes blandas como lo es una capsulotomía anterior (que realizamos) o tenotomías de los grupos musculares retraídos en flexión. ${ }^{12}$

La amputación supracondílea es una entidad secundaria a diferentes etiologías (en menor cantidad son traumáticas); la mayoría involucra una sola extremidad, para nosotros es el segundo caso reportado con amputación bilateral y fractura de cuello femoral, en el cual se decidió el tratamiento con ATC con muy buenos resultados a corto plazo, un buen pronóstico y readaptación a sus actividades diarias de nuestro paciente.

Si bien se tienen dificultades en su manipulación y se describen varias técnicas para ayudar con este problema, optamos por tracción manual, así como la correcta colocación del arco de fluoroscopía que nos ayudará con obtención de imágenes anteroposterior y lateral adecuadas para la colocación de la prótesis.

Bibliografía

1. Amanatullah DF, Trousdale RT, Sierra RJ. Total hip arthroplasty after lower extremity amputation. Orthopedics. 2015; 38(5): e394-e40.

2. Masmoudi M, Rbai H. Primary total hip replacement for a femoral neck fracture in a below-knee amputee. J Orthop Case Rep. 2016; 6(3): 63-6.

3. Faour O, Navarro E. Artroplastía total de cadera en paciente con amputación suprageniana ipsilateral. Caso clínico y revisión de la literatura. Revista Española de Cirugía Osteoarticular. 2011; 46: 247.

4. Rethnam U, Yesupalan RS, Shoaib A, Ratnam TK. Hip fracture fixation in a patient with below-knee amputation presents a surgical dilemma: a case report. J Med Case Rep. 2008; 2: 296.

5. Mak J, Solomon M, Faux S, Ipsilateral total hip arthroplasty in a dysvascular below-knee amputee for advanced hip osteoarthritis: A case report review of the literatura. Prosthet Orthot Int. 2008; 32(2): 155-9.

6. Pekmezci M, Nunley R. Technique for total hip arthroplasty in a patient with through-knee amputation. J Arthroplasty. 2010; 25(4): 659e1-3.

7. Kandel L, Hernandez M. Bipolar hip hemiarthroplasty in a patient with an above knee amputation: a case report. J Orthop Surg Res. 2009; 4: 30.

8. Bong MR, Kaplan KM. Total hip arthroplasty in a patient with contralateral hemipelvectomy. J Arthroplasty. 2006; 21(5): 762-4. 
9. Salai M, Chechik A. Total hip arthroplasty in patients with below-knee amputations. J Arthroplasty. 2000; 15(8): 999-1002.

10. Deathe AB, Wolfe DL, Devlin M, Hebert JS, Miller WC, Pallaveshi L. Selection of outcome measures in lower extremity amputation rehabilitation: ICF activities. Disabil Rehabil. 2009; 31(18): 1455-73.

11. Aqil A, Desai A. A simple technique to position patients with bilateral above-knee amputations for operative fixation of intertrochanteric fractures of the femur: a case report. J Med Case Rep. 2010; 4: 390.
12. Malagelada F, Coll RM. Total hip replacement in an ipsilateral above-the-knee amputation: surgical technique, rehabilitation, and review of the literature. Int J Low Extrem Wounds. 2013; 12(1): 3943.

13. Berg AJ, Bhatia C. Neck of femur fracture fixation in a bilateral amputee: an uncommon condition requiring an improvised fracture table positioning technique. BMJ Case Rep. 2014; 2014. 